\title{
Magnetic Susceptibility of Coupled Double GaAs Quantum Dot in Magnetic Fields
}

\author{
M.K. ELSAid* AND E. HiJAZ \\ Physics Department, Faculty of Science, An-Najah National University, Nablus, West Bank, Palestine \\ (Received August 2, 2016; in final form March 22, 2017)

\begin{abstract}
We calculate the magnetic susceptibility of two interacting electrons confined in a coupled double quantum dot presented in a magnetic field by solving the relative Hamiltonian using the combined variational and exact diagonalization methods. We have investigated the dependence of the magnetic susceptibility on temperature, magnetic field strength, confining frequency, and barrier height. The singlet-triplet transitions in the ground state of the quantum dot spectra and the corresponding jumps in the magnetic susceptibility curves have been shown. The comparisons show that our results are in very good agreement with reported works.
\end{abstract}

DOI: 10.12693/APhysPolA.131.1491

PACS/topics: magnetic susceptibility, variational method, exact diagonalization, double quantum dots

\section{Introduction}

Quantum dots (QDs), or artificial atoms, had been the subject of interest research due to their physical properties and great potential device applications such as quantum dot lasers, solar cells, single electron transistors, and quantum computers [1-5]. The application of a magnetic field perpendicular to the dot plane will introduce an additional structure on the energy levels and correlation effects of the interacting electrons confined in a quantum dot. Different approaches have been used to solve the two electrons QD Hamiltonian, including the effect of an applied magnetic field, to obtain the eigenenergies and eigenstates of the QD-system. Wagner et al. [6] studied this interesting QD system and predicted the oscillations between spin-singlet (S) and spin-triplet (T) ground states. Taut [7] managed to obtain the exact analytical results for the energy spectrum of two interacting electrons through a Coulomb potential, confined in a QD, just for particular values of the magnetic field strength. In Refs. [8, 9] the authors had solved the QD-Hamiltonian by variational method and obtained the ground state energies for various values of magnetic field $\omega_{c}$, and confined frequency $\omega_{0}$. In addition, they had performed exact numerical diagonalization for the helium QD-Hamiltonian and obtained the energy spectra for zero and finite values of magnetic field strength. Kandemir $[10,11]$ found the closed form solution for this QD Hamiltonian and the corresponding eigenstates for particular values of the magnetic field strength and confinement frequencies. Elsaid [12-16] used the dimensional expansion technique, in different works, to solve the QD-Hamiltonian and obtain the energies of the two interacting electrons for any arbitrary ratio of Coulomb to confinement energies and gave an explanation to the level crossings.

*corresponding author; e-mail: mkelsaid@najah.edu
Maksym and Chakraborty [17] implemented the diagonalization method to obtain the eigenenergies of interacting electrons in a magnetic field and show the transitions in the angular momentum of the ground states. They also had calculated the heat capacity curve for both interacting and non-interacting confined electrons in the QD presented in a magnetic field. The interacting model shows very different behavior from non-interacting electrons, and the oscillations in these magnetic and thermodynamic quantities like magnetization $\mathcal{M}$ and heat capacity $C_{v}$ are attributed to the spin singlet-triplet transitions in the ground state spectra of the quantum dot. De Groote et al. [18] also computed the magnetization, susceptibility and heat capacity of helium like confined QDs and obtained the additional structure in magnetization. In a detailed study, Nguyen and Peeters [19] considered the QD helium in the presence of a single magnetic ion and applied magnetic field taking into account the electron-electron correlation in many quantum dots. They showed the dependence of these thermal and magnetic quantities $C_{v}, \mathcal{M}$, and $\chi$ on the strength of the magnetic field, confinement frequency, magnetic ion position, and temperature. They observed that the cusps in the energy levels show up as peaks in the heat capacity and magnetization.

In Ref. [20], the authors used the static fluctuation approximation (SFA) to study the thermodynamic properties of two-dimensional GaAs/AlGaAs parabolic QD in a magnetic field. Boyacioglu and Chatterjee [21] studied the magnetic properties of a single quantum dot confined with a Gaussian potential model. They observed that the magnetization curve shows peaks structure at low temperature. Helle et al. [22] computed the magnetization of a two-electron lateral quantum dot molecule (QDM) in a high magnetic field at zero temperature and the results show the oscillation and smooth behavior in the magnetization curve for both, interacting and non-interacting confined electrons, respectively.

In an experimental work [23], the magnetization of electrons in $\mathrm{GaAs} / \mathrm{AlGaAs}$ semiconductor $\mathrm{QD}$ as 
function of applied magnetic field at low temperature $0.3 \mathrm{~K}$ was measured. They observed oscillations in the magnetization. To reproduce the experimental results of the magnetization, they found that the electron-electron interaction should be taken into account in the theoretical model of the QD magnetization. Furthermore, the density functional method (DFT) was used to investigate the magnetization of a rectangular QD in the applied external magnetic field [24]. Climente et al. studied the effect of Coulomb interaction on the magnetization of quantum dot with one and two interacting electrons [25].

Very recently, Avetisyan et al. [26] studied the magnetization of anisotropic QD in the presence of the Rashba spin-orbit interaction for three interacting electrons in the dot. In Ref. [27], the authors have studied the electron-electron correlations in many-electron single quantum dot (SQD) confined by parabolic potential in the presence of single magnetic ion and perpendicular magnetic field. They have obtained the energies and have studied the thermodynamic quantities such as heat capacity, the magnetization and the susceptibility. They have found that the cusps in the curves of these heat quantities are due to the transitions in the angular momentum of the energy levels of the QD. Dybalski and Hawrylak [28] also studied the electronic properties of two electrons in a strongly coupled double quantum dot (DQD). They have analyzed the singlet-triplet gap with the barrier height potential and with an external magnetic field. The authors solved the full Hamiltonian of DQD by using the variational method to calculate separately the energy matrix elements of SQD term and barrier potential term. Next, the exact diagonalization method is implemented to compute the corresponding energies of the full DQD Hamiltonian. The authors of Ref. [29] used the Huns-Mulliken molecular orbit approach to calculate the energy levels of two electrons confined in two laterally coupled quantum dots, under the influence of an applied magnetic field. They showed that the jumps of the magnetization are due to the singlet-triplet transitions in the energy levels.

The purpose of this work is to calculate the magnetic susceptibility as a thermodynamic quantity for a coupled double quantum dots presented in a magnetic field taking into account the electron-electron Coulomb interaction term. Since, the eigenvalues of the electrons in the DQD are necessary input quantities to calculate the statistical average energies of the DQD system, to achieve this target we have implemented the variation and exact diagonalization methods to obtain the desired eigenenergies. The computed eigenenergies spectra were used to study theoretically the dependence of the magnetic susceptibility curve of the coupled DQD as a function of magnetic field strength, confining frequency barrier height and temperature.

The rest of this paper is organized as follows: the Hamiltonian theory and computation techniques of two interacting electrons in DQD are presented in Sect. 2. In Sect. 3, we show how to calculate the magnetic susceptibility from the mean energy expression. Final section will be devoted for numerical results and conclusion.

\section{The double quantum dots Hamiltonian}

Consider two interacting electrons inside a double quantum dots confined by a parabolic potential of strength $\omega_{0}$ under the effect of an applied uniform magnetic field of strength $\omega_{c}$, taken to be along $z$-direction, in addition to a coupled Gaussian barrier of width $\Delta$ and height $V_{0}$. This model can be characterized by the Hamiltonian $\left(H_{\mathrm{DQD}}\right)$ :

$$
\begin{gathered}
H_{\mathrm{DQD}}=\sum_{j=1}^{2}\left[\frac{1}{2 m^{*}}\left(\boldsymbol{p}\left(\boldsymbol{r}_{\boldsymbol{j}}\right)+\frac{e}{c} \boldsymbol{A}\left(\boldsymbol{r}_{\boldsymbol{j}}\right)\right)^{2}+\frac{1}{2} m^{*} \omega_{0}^{2} r_{j}^{2}\right] \\
+\frac{e^{2}}{\varepsilon_{0}\left|r_{1}-r_{2}\right|}+V_{0}\left(\mathrm{e}^{-x_{1}^{2} / \Delta^{2}}+\mathrm{e}^{-x_{2}^{2} / \Delta^{2}}\right),
\end{gathered}
$$

where $r_{j}$ and $p\left(r_{j}\right)$ are the position and momentum of the electron inside the QD. In addition, $x_{1}$ and $x_{2}$ represent the position of each quantum dot along the $x$-direction.

$H_{\text {DQD }}$ can be considered as the sum of the single quantum dot Hamiltonian $\left(H_{\mathrm{SQD}}\right)$ and the potential barrier term $V_{b}=V_{0}\left(\mathrm{e}^{-x_{1}^{2} / \Delta^{2}}+\mathrm{e}^{-x_{2}^{2} / \Delta^{2}}\right)$ as follows:

$$
H_{\mathrm{DQD}}=H_{\mathrm{SQD}}+V_{b} \text {. }
$$

It is obvious that the single quantum dot Hamiltonian, $H_{\mathrm{SQD}}$ can be obtained from Eq. (2.2) by making the barrier potential term vanish, $V_{b}=0$. Using the standard coordinate transformation and adopting the symmetric gauge, the single quantum dot Hamiltonian can be separated into a center of mass Hamiltonian, $H_{c m}$, and a relative Hamiltonian part $H_{r}$ as shown below:

$$
\begin{aligned}
& H_{c m}=\frac{1}{2 M}\left(P_{R}+\frac{Q}{c} A(R)\right)^{2}+\frac{1}{2} M \omega_{0}^{2} R^{2}, \\
& H_{r}=\frac{1}{2 \mu}\left(p_{r}+\frac{q}{c} A(r)\right)^{2}+\frac{1}{2} \mu \omega_{0}^{2} r^{2}+\frac{e^{2}}{\epsilon|r|},
\end{aligned}
$$

where $M$ is the total mass $=2 m, Q$ is the total charge $=2 e, \mu$ is reduced mass $=\frac{m}{2}$, and $q$ is the reduced charge $=\frac{e}{2}$.

The corresponding energy of the $H_{\mathrm{SQD}} \mathrm{Eq} .(2.2)$ is

$$
E_{\mathrm{SQD}}=E_{c m}+E_{r} \text {. }
$$

The center of mass Hamiltonian (2.3) has the harmonic oscillator form with well-known fully analytical solution for wave function and energy given, respectively, as,

$$
\begin{gathered}
\psi_{n_{c m}, m_{c m}}(R)=(-1)^{n_{c m}} \frac{\lambda^{\left|m_{c m}+1\right|}}{\sqrt{\pi}} \sqrt{\frac{n_{c m} !}{\left(n_{c m}+\left|m_{c m}\right|\right) !}} \\
\times \mathrm{e}^{-\lambda^{2} R^{2} / 2} R^{\left|m_{c m}\right|} L_{n_{c m}}^{\left|m_{c m}\right|}\left(\lambda^{2} R^{2}\right) \mathrm{e}^{\mathrm{i} m_{c m} \phi}, \quad(2.6) \\
E_{n_{c m}, m_{c m}}=\left(2 n_{c m}+\left|m_{c m}\right|+1\right) \hbar \omega+m_{c m} \frac{\hbar \omega_{c}}{2},(2.7)
\end{gathered}
$$

where $\lambda=\sqrt{\frac{m^{*} \omega}{\hbar}}$, and $\omega=\sqrt{\omega_{0}^{2}+\frac{\omega_{c}^{2}}{4}}$ are constants. $n_{c m}, m_{c m}$ are the radial and azimuthal quantum numbers, respectively, and $L_{n}^{m}$ is the associate Laguerre polynomials [8]. 
The relative Hamiltonian part Eq. (2.4) does not have an analytical solution for all ranges of $\omega_{0}$ and $\omega_{c}$, due to the existence of both Coulomb and parabolic terms, so the variational method has been used as an accurate method to get the energy spectra $\left(E_{r}\right)$ for the relative Hamiltonian in terms of a variational parameter. The adopted variational wave function and the corresponding energy equation are given in Appendix A.

The calculations of the energy matrix elements of the $V_{b}$-barrier term using the variational method were given in Ref. [28] and will not be repeated here. The combined terms of the single quantum dot Hamiltonian energy and barrier energy matrix elements are diagonalized to give the full matrix elements of the DQD Hamiltonian [28]. We would like to emphasize again that our aim in this work is to investigate the effect of barrier height and confining frequency on the magnetic susceptibility of the DQD, so only the essential steps which lead to the main eigenenergy equation of the full DQD Hamiltonian will be shown in Appendix B.

\section{Magnetic susceptibility of DQD}

We have computed energies of the DQD system as essential input data to calculate the magnetic susceptibility of the DQD. The magnetic susceptibility of the DQD system is evaluated as the second magnetic field derivative of the mean energy of the DQD:

$$
\chi\left(T, B, \omega_{0}, V_{0}, \Delta\right)=-\frac{\partial^{2}\left\langle E\left(T, B, \omega_{0}\right)\right\rangle}{\partial^{2} B},
$$

where the statistical average energy is calculated as

$$
\left\langle E\left(T, B, \omega_{0}, V_{0}, \Delta\right)\right\rangle=\frac{\sum_{\alpha=1}^{N} E_{\alpha} \mathrm{e}^{-E_{\alpha} / k_{\mathrm{B}} T}}{\operatorname{sum}_{\alpha=1}^{N} \mathrm{e}^{-E_{\alpha} / k_{\mathrm{B}} T}},
$$

where $\alpha$ denotes the quantum numbers of the coupled quantum dots energy states obtained by diagonalizing the DQD full Hamiltonian (Eq. (B2)). We use Eqs. (2.8) and (2.9) to investigate the dependence of the magnetic susceptibility of the double quantum dots on very rich and tunable physical parameters, namely: the temperature $(T)$, magnetic field strength $\left(\omega_{c}\right)$, confining potential $\left(\omega_{0}\right)$, barrier height $\left(V_{0}\right)$ and barrier width $(\Delta)$.

\section{Results and discussions}

The computed results for two interacting electrons in double quantum dots made from GaAs material $\left(m^{*}=\right.$ $0.067 m_{e}, R^{*}=5.825 \mathrm{meV}$ ) are presented in Figs. 1 to 6 . Figure 1a shows the calculated eigenenergy spectra of DQD for angular momentum $m=0,1$ and 2 as a function of magnetic field strength $\omega_{c}=0.0$ to 4.0 , confining frequencies $\omega_{0}=\frac{2}{3} R^{*}$ and barrier heights $V_{0}=1 R^{*}$. The calculated energy results are in excellent agreement with previous reported work [28]. In Fig. $1 \mathrm{~b}$ we have plotted also the computed energy results of this work against the strength of the magnetic field for $\omega_{0}=\frac{2}{3} R^{*}$, $\Delta=0.5 R^{*}, V_{0}=1 R^{*}$ for small range of $\omega_{c}=\left\{0,1 R^{*}\right\}$ to show the level crossing more clearly. The energy level plot
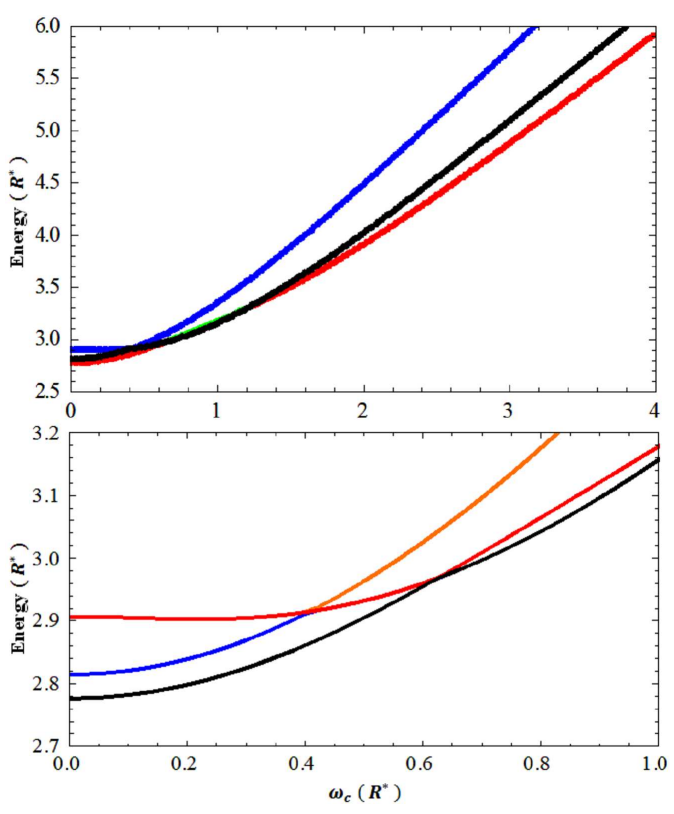

Fig. 1. The computed energy spectra of two interacting electrons in double quantum dots against the strength of the magnetic field for $\omega_{0}=\frac{2}{3} R^{*}, \Delta=0.5 R^{*}$, $V_{0}=1 R^{*}$, angular momentum $m=0,1,2$ and for the range of $\omega_{c}=\left\{0,4 R^{*}\right\}$ (a) and $\left\{0,1 R^{*}\right\}$ (b).
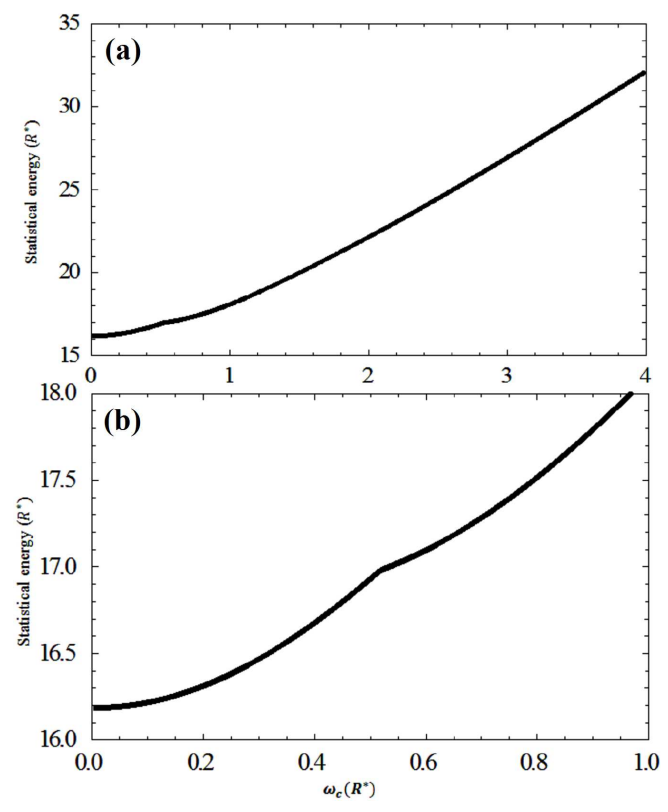

Fig. 2. The statistical energy of two interacting electrons in double quantum dots against the strength of the magnetic field for $\omega_{0}=\frac{2}{3} R^{*}, \Delta=0.5 R^{*}, V_{0}=1 R^{*}$. The curve in part (b)shows a cusp at $\omega_{c}=0.5 R^{*}$.

shows obviously the transition in the angular momentum of the ground state of the DQD system as the magnetic field strength increases. The origin of these transitions is due to the effect of the Coulomb interaction energy in the QD systems [22]. The singlet-triplet transitions in the angular momentum of the DQD system manifest themselves as cusps in the magnetic susceptibility curve 


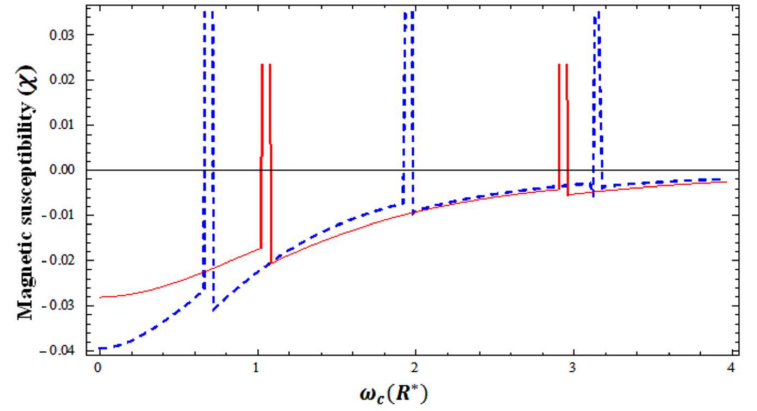

Fig. 3. The magnetic susceptibility $\chi$ at $T=0.01 \mathrm{~K}$, of two interacting electrons in SQD against the magnetic field strength for different confinements: $\omega_{0}=\frac{2}{3} R^{*}$ (dashed line) and $\omega_{0}=0.9 R^{*}$ (solid line).

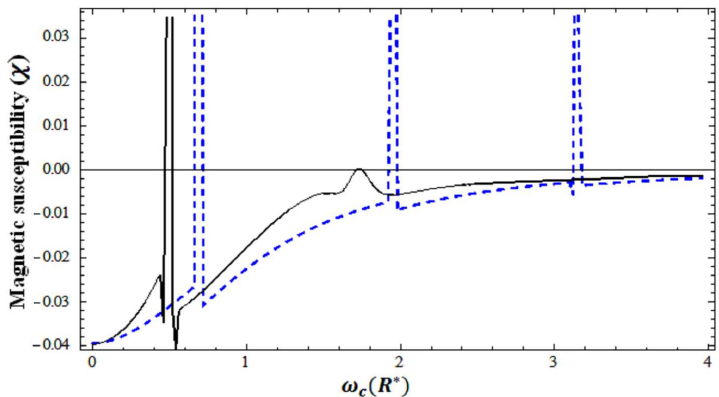

Fig. 4. Comparison between the magnetic susceptibility $\chi$ curves at $T=0.01 \mathrm{~K}$ of the two interacting electrons in SQD (dashed line) with confinement $\omega_{0}=\frac{2}{3} R^{*}$ and DQD (solid line) for $\omega_{0}=\frac{2}{3} R^{*}, \Delta=0.5 R^{*}$, $V_{0}=1 R^{*}$ against the magnetic field strength.

of the DQD. Our energy spectra results show very good agreement compared with corresponding one in Fig. 3 of Dybalski's results where the authors used the variational method to solve the DQD Hamiltonian [28]. In addition we have plotted the statistical energy against the strength of magnetic field for wide range and small range of $\omega_{c}$ in Fig. 2a and b, respectively. The figures show clearly the origin of the cusps in the statistical energy curves that causes the corresponding cusps in the magnetic susceptibility curve of the DQD.

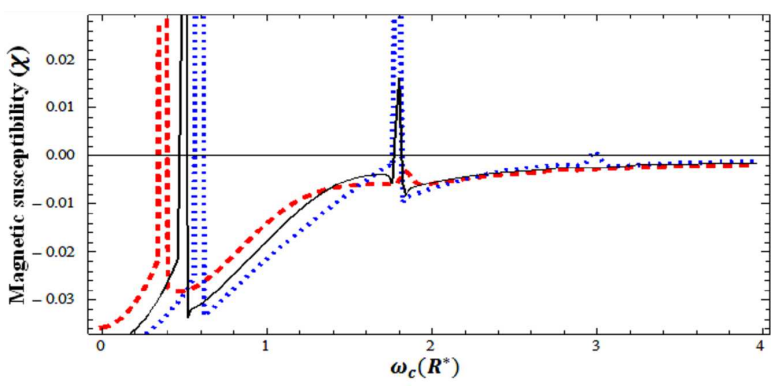

Fig. 5. The magnetic susceptibility $\chi$ at $T=0.01 \mathrm{~K}$, of the two interacting electrons in DQD against the magnetic field strength for $\omega_{0}=\frac{2}{3} R^{*}, \Delta=0.5 R^{*}$. Dotted curve: $V_{0}=0.5 R^{*}$, solid curve: $V_{0}=R^{*}$, dashed curve: $V_{0}=1.5 R^{*}$.
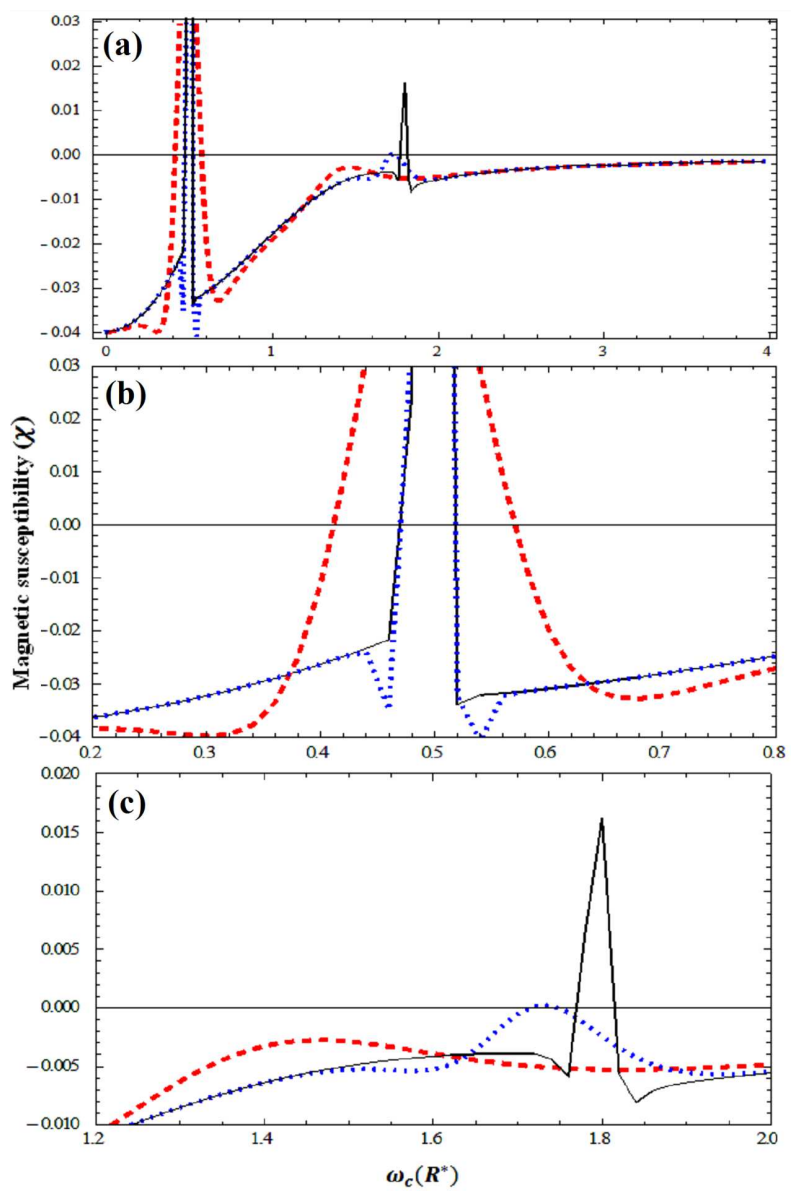

Fig. 6. (a) The magnetic susceptibility $\chi$ of the two interacting electrons in DQD against the magnetic field strength for $\omega_{0}=\frac{2}{3} R^{*}, \Delta=0.5 R^{*}, V_{0}=R^{*}$, at $T=$ $0.01 \mathrm{~K}$ (solid curve), at $T=0.1 \mathrm{~K}$ (dotted curve), at $T=1 \mathrm{~K}$ (dashed curve). Part (b) shows the presence of first cusp, part (c) shows the presence of second cusp.

In Fig. 3, we have investigated the effect of the parabolic confinement strength, $\omega_{0}$, on the magnetic susceptibility curves for SQD. The magnetic susceptibility curves show that: as the confinement strength increases from $\omega_{0}=\frac{2}{3}=0.667 R^{*}$ (dashed curve) to $\omega_{0}=0.9 R^{*}$ (solid curve), the number of peaks in the susceptibility curves decreases from 3 to 2 peaks and shifts to the right, towards a high magnetic field strength. This peak behavior in the magnetic susceptibility curve can be explained as follows. As the parabolic confinement $\omega_{0}$ increases, the confinement energy of the electrons in the QD increases also and in this case a magnetic field with high strength is required to make the transition.

In Fig. 4, we have compared the magnetic susceptibility curves for both DQD (solid curve) and SQD (dashed curve). The comparison shows that the number of peaks reduced from three peaks in the SQD to only two peaks in the DQD. This peak reduction behavior is attributed to the enhancement in the confinement of the electron energies in the DQD as the height of the potential barrier $V_{0}$ increases. We have used a similar argument to 
understand the behavior of magnetic susceptibility for SQD case presented in Fig. 3.

Furthermore, we have investigated the effect of the barrier height $V_{0}$ on the magnetic susceptibility curve. We have plotted in Fig. 5 the magnetic susceptibility curves calculated at different barrier heights namely, $V_{0}=0.5$ (dotted curve), 1.0 (solid curve) and 1.5 (dashed curve). The comparison clearly shows the gradual shift of the magnetic susceptibility jumps to higher magnetic field as the barrier height decreases.

In addition we have studied the effect of temperature on the magnetic susceptibility curves of DQD system as function of the magnetic field strengths with different ranges calculated at three different temperatures $T=0.01 \mathrm{~K}$ (solid curve), $0.1 \mathrm{~K}$ (dotted curve) and $1 \mathrm{~K}$ (dashed curve) as shown in Fig. 6ac. We have seen from the figure that the temperature shows an effect on the cusps of the magnetization curves. To show this effect more clearly we have focused in Fig. 6b and c, on the first and second magnetization cusps, respectively. We have noticed from the figures that the high-field magnetic susceptibility peak shifts to lower magnetic fields with increase of the temperature.

In conclusion, we have applied the combined exact diagonalization and variational calculation methods to solve the Hamiltonian for two interacting electrons confined in a double-quantum dot presented in a magnetic field. We have investigated the dependence of the magnetization of the DQD on the magnetic field strength, confining frequency, barrier width, barrier height, and temperature.

\section{Appendix A: Energy calculations of SQD by variation method}

This appendix gives the main expressions that have been used to compute the eigenenergy expressions of the DQD.

The adopted one parameter variational wave function is taken as

$$
\psi(r)=\sqrt[4]{\alpha} \frac{u_{m}(\rho) \mathrm{e}^{\mathrm{i} m \phi}}{\sqrt{2 \pi \rho}},
$$

where

$$
\begin{aligned}
& u_{m}(\rho)=C_{m} \rho^{1 / 2+|m|}(1+\beta \rho) \mathrm{e}^{-\left(\frac{\rho^{2}}{2}\right)}, \\
& \rho=\sqrt{\alpha} r, \text { and } \alpha=\frac{1}{4} \sqrt{\frac{\omega_{c}^{2}}{4}+\omega_{0}^{2}},
\end{aligned}
$$

with normalization constant $C_{m}$, which can be expressed in terms of standard gamma function $\Gamma(x)$, angular momentum $m$, and parameters $\alpha$, and $\beta_{\min }[28]$.

We proceed to obtain the energies of the relative part of the single quantum dot Hamiltonian by calculating the energy matrix elements $E_{r}=\left\langle\psi\left|H_{r}\right| \psi\right\rangle$ as

$$
E_{r}(\beta)=-\frac{1}{2} m \omega_{c}+2 \alpha \frac{a+b \beta+c \beta^{2}}{d+e \beta+f \beta^{2}},
$$

where $a, b$ and $c$ are constants in terms of quantum numbers $m$ and $\alpha$.
The energy eigenvalues of $H_{r}$ can be found by minimizing the energy formula Eq. (A4) with respect to the variational parameter $\beta$ to give

$$
\begin{aligned}
& \beta_{\min , m}= \\
& \frac{2 c d-2 a f-\sqrt{(2 c d-2 a f)^{2}-4(b d-a e)(c e-b f)}}{2(-c e+b f)} .
\end{aligned}
$$

So, the energy expression of the SQD Hamiltonian in terms of the variational parameter, $\beta_{\text {min }}$, which satisfies the minimization condition, is

$$
E_{r}\left(\beta_{\min }\right)=-\frac{1}{2} m \omega_{c}+2 \alpha \frac{a+b \beta_{\min }+c \beta_{\min }^{2}}{d+e \beta_{\min }+f \beta_{\min }^{2}} .
$$

\section{Appendix B: Energy calculations of double quantum dot}

To compute the full energy spectra of the DQD system we have set $V_{0}>0$ in the Hamiltonian model Eq. (2.1), so the potential of the barrier is

$$
V_{b}=V_{0}\left(\mathrm{e}^{-x_{1}^{2} / \Delta^{2}}+\mathrm{e}^{-x_{2}^{2} / \Delta^{2}}\right) .
$$

The matrix element of the DQD can be evaluated in terms of elliptic functions, angular quantum number $m$, barrier height $V_{0}$ and barrier width $\Delta$. The combined terms of the single quantum dot energy $E_{m}\left(\beta_{m}\right)$ and barrier energy matrix elements will give the full matrix elements of the DQD Hamiltonian. We implemented the diagonalization technique to obtain the eigenergies of the $H_{\mathrm{DQD}}$ :

$$
H_{m, n}=\left(E_{m}\left(\beta_{m}\right)+E_{c m}\right) \delta_{m, n}+\left\langle u_{m}\right\rangle,
$$

where $E_{c m}$ is the center of mass Hamiltonian as defined previously in Eq. (2.7).

\section{References}

[1] R.C. Ashoori, H.L. Stormer, J.S. Weiner, L.N. Pfeiffer, K.W. Baldwin, K.W. West, Phys. Rev. Lett. 71, 613 (1993).

[2] C. Ciftja, Phys. Scr. 72, 058302 (2013).

[3] M.A. Kastner, Rev. Mod. Phys. 64, 849 (1992).

[4] D. Loss, D.P. Divincenzo, Phys. Rev. A 57, 120 (1998).

[5] G. Burkard, D. Loss, D.P. Divincenzo, Phys. Rev. B 59, 2070 (1999).

[6] M. Wagner, M.U. Merkt, A.V. Chaplik, Phys. Rev. B 45, 1951 (1992).

[7] M. Taut, J. Phys. A Math. Gen. 27, 1045 (1994).

[8] C. Ciftja, A.A. Kumar, Phys. Rev. B 70, 205326 (2004).

[9] O. Ciftja, M. Golam Faruk, Phys. Rev. B 72, 205334 (2005).

[10] B.S. Kandemir, Phys. Rev. B 72, 165350 (2005).

[11] B.S. Kandemir, J. Math. Phys. 46, 032110 (2005).

[12] M. Elsaid, Phys. Rev. B 61, 13026 (2000).

[13] M. Elsaid, Semiconductor Sci. Technol. 10, 1310 (1995). 
[14] M. Elsaid, Superlatt. Microstruct. 23, 1237 (1998).

[15] M. Elsaid, M.A. Al-Naafa, S. Zugail, J. Comput. Theor. Nanosci. 5, 677 (2008).

[16] M. Elsaid, Phys. Status Solidi B 184, 385 (1994).

[17] P.A. Maksym, T. Chakraborty, Phys. Rev. Lett. 65, 108 (1990).

[18] J.J.S. De Groote, J.E.M. Hornos, A.V. Chaplik, Phys. Rev. B 46, 12773 (1992).

[19] N.T.T. Nguyen, F.M. Peeters, Phys. Rev. B 78, 045321 (2008).

[20] F.S. Nammas, A.S. Sandouqa, H.B. Ghassib, M.K. Al Sugheir, Physica B 406, 4671 (2011).

[21] B. Boyacioglu, A. Chatterjee, J. Appl. Phys. 112, 083514 (2012).

[22] M. Helle, A. Harju, R.M. Nieminen, Phys. Rev. B 72, 205329 (2005).
[23] M.P. Schwarz, D. Grundler, M. Wilde, Ch. Heyn, D. Heitmann, J. Appl. Phys. 91, 6875 (2002).

[24] E. Räsänen, H. Saarikoski, V.N. Stavrou, A. Harju, M.J. Puska, R.M. Nieminen, Phys. Rev. B 67, 235307 (2003).

[25] J.I. Climente, J. Planelles, J.L. Movilla, Phys. Rev. B 70, 081301 (2004).

[26] S. Avetisyan, T. Chakraborty, P. Pietiläinen, Physica E 81, 334 (2016).

[27] N.T.T. Nguyen, S. Das Sarma, Phys. Rev. B 83, 235322 (2011).

[28] W. Dybalski, P. Hawrylak, Phys. Rev. B 72, 205432 (2005).

[29] N. Rachid, E. Ben Salem, R. Jaziri, S. Bennaceu, Physica E 41, 568 (2009). 